\title{
Evolution des concepts concernant le pilotage des irrigations
}

\author{
Jean-Marc Deumier \\ Institut Technique des Céréales et des Fourrages \\ 6, Chemin de la Côte Vieille, 31450 Baziège \\ Paul Peyremorte \\ Société du Canal de Provence et d'Aménagement de la Région Provençale \\ B.P. 100 Le Tholonet, 13603 Aix-en-Provence Cédex \\ avec la collaboration de :

\section{Pascal Leroy} \\ Institut National de la Recherche Agronomique \\ Laboratoire d'Economie et de Sociologie Rurales de Paris-Grignon \\ Claude Jacquin et Alain Bouthier \\ Institut Technique des Céréales et des Fourrages
}

Bien des raisons conduisent au progrès de la maitrise des arrosages.

Les circonstances climatiques que nous venons de connaître, au cours des dernières années, ont d'ailleurs beaucoup aidé à mieux apprécier la valeur de l'eau :

- la prépondérance des usages urbains et industriels est à présent mieux connue et chacun a pu apprécier l'intérêt majeur que représente la garantie de ressource qui est aussi celle du profit que l'on peut espérer de l'irrigation ;

- également, la pénurie a permis de mieux considérer l'importance primordiale du contexte hydrique des sols, notamment des couches profondes, sur les besoins effectifs d'arrosage.

Par ailleurs, la nécessité de respecter notre environnement, d'une part, les conséquences économiques des récentes dispositions politiques de la CEE concernant les gran- des productions agricoles, d'autre part, vont augmenter la nécessité d'optimiser les moyens de production. Pour l'irrigation des grandes cultures la conduite des arrosages sera davantage tributaire de la gestion de l'ensemble des moyens d'irrigation que des besoins instantanés des plantes : à la notion d'ETM, très utilisée jusqu'alors pour éviter les abus d'irrigation, il faut substituer celle d'ET optimale qui peut être plus dépendante de considérations économiques que techniques.

Les progrès de l'électronique appliqués à la mesure d'indicateurs d'état hydrique du sol, voire du végétal, ouvrent des perspectives très intéressantes pour apporter, aux aspects stratégiques que nous venons d'énoncer, les compléments indispensables, au plan tactique, pour adapter la conduite des irrigations selon les circonstances particulières de lieu ou de temps.

\section{Evolution of water supply management concepts}

Irrigation context diversity leads to water supply management modalities due to very different concepts, mainly dependent on constraints. For great margin crops, main object is to guarantee economic performance often linked to product quality; in this case we note development of automatic water management tools based on soil or plant hydric status. For large scale growing, irrigation cost is very important compared to other costs; so water supply management depends on equipment and hydraulic constraints. 


\section{Conduite des arrosages en condition de faible coût relatif de l'eau utilisée}

Dès lors que le coût de l'irrigation ne représente qu'une faible fraction du produit brut assuré par la culture irriguée l'optimum économique se rapproche de l'optimum technique. L'objectif du pilotage, dans de telles circonstances, est de garantir la satisfaction optimale des besoins en eau de la culture tout en réduisant les contraintes de réalisation des arrosages.

Selon le mode de distribution utilisé la fréquence des interventions incite à une plus ou moins grande automatisation des apports d'eau. En aspersion classique, il s'agit le plus souvent d'assurer une couverture intégrale en équipement d'arrosage pour n'avoir qu'à sélectionner le secteur à irriguer. En apports localisés la fréquence des interventions nécessaires conduit à automatiser également les cycles d'arrosage à une fréquence parfois pluri-quotidienne.

Cependant l'automatisation des apports d'eau, même lorsqu'elle résulte d'appareillages parfois complexes, n'est pas une garantie de parfaite adéquation des apports aux besoins exacts de la culture.

\section{Trop c'est trop!}

Le risque est grand de pratiquer des apports excessifs pour le moins inefficaces, souvent néfastes et toujours polluants.

En aspersion certains indicateurs, ne serait-ce que celui concernant la portance du sol, peuvent constituer un repère élémentaire pour éviter ce type d'abus. L'emploi de capteurs, permettant d'apprécier l'état hydrique du sol (par exemple les mesures tensiométriques) malgré les contraintes de leur utilisation, donne des informations beaucoup plus intéressantes par exemple pour choisir fréquence et dose d'apport.

En irrigation localisée, particulièrement avec le goutte à goutte, il est indispensable de s'assurer de l'efficacité de l'eau apportée. Cela concerne le bon ajustement de la dose périodique totale mais aussi son fractionnement. Le risque avec ce mode d'apport est tout autant celui de l'excès que celui du manque d'eau. Cependant l'irrigation localisée est la seule méthode qui permette d'assurer un rationnement optimal de l'alimentation en eau des cultures qui ne s'accommodent pas d'une alimentation à l'ETM.

Or un grand nombre de productions fruitières se trouvent dans ce cas : qualitativement pour beaucoup d'entreelles mais aussi quantitativement pour certaines, les meilleurs résultats ne sont pas obtenus avec une alimentation ad libitum.

\section{La nécessité de savoir ce qui se passe dans le sol}

Nous venons de mentionner l'intérêt des mesures in situ pour améliorer la conduite des arrosages. Paradoxalement, on critique souvent ce type d'investigation alors que l'on accepte sans réserve des informations indépendantes du système que l'on prétend maîtriser (PEYREMORTE P., 1984). L'estimation des besoins d'arrosage basés sur le niveau potentiel d'évapotranspiration bénéficie à cet égard d'un a priori abusivement favorable. Comment peut-on en arriver jusqu'à confondre parfois besoin en eau des cultures et besoin d'irrigation alors que ce dernier, tout particulièrement en apports localisés, dépend davantage des circonstances de sol (de la contribution des couches profondes, de l'efficacité des pluies) que des variations interannuelles de l'ETP périodique (PEYREMORTE P., 1990).

\section{Du progrès de l'informatique à celui de la maîtrise des arrosages}

L'amélioration des performances des composants électroniques et la réduction de leur coût permet de disposer d'équipements très peu exigeants en énergie et vulgarisables à grande échelle.

D'ores et déjà existent en France deux types d'automate capables de mesurer en permanence, à partir d'un nombre de capteurs suffisants pour assurer la représentativité des informations, soit l'état hydrique du sol, soit la réponse des cultures à plus ou moins grande satisfaction des besoins en eau.

Le premier désigné HUMICRO 2000 mesure la tension de l'eau dans le sol à partir de 8 capteurs et en présente quotidiennement les résultats sous forme de graphiques. L'analyse des courbes permet de juger du bon choix de la fréquence et de la dose d'arrosage. Cet appareil peut en outre assurer directement le pilotage des apports lorsque leur fréquence est inférieure à une semaine; a fortiori quand il s'agit d'apports pluriquotidiens comme cela se justifie parfois en apports localisés (PEYREMORTE P., CHOL P., 1992).

Par ailleurs, ce matériel permettra de beaucoup simplifier la prise d'information des mesures tensiométriques en vue de mieux conduire les arrosages ; méthode qui a largement fait ses preuves en pilotage non automatique, mais qui exigeait jusqu'alors des interventions contraignantes.

Le deuxième, mis au point par l'I.N.R.A., désigné PEPISTA, mesure la variation micrométrique des organes végétaux (tiges, fruits) qui est un bon indicateur de l'état de satisfaction des besoins en eau de la culture. Cet outil permet un diagnostic précis du comportement des plantes. Il peut aussi comporter un logiciel de commande des arrosages (Huguet J.G., 1992).

\section{La conduite de l'irrigation des grandes cultures}

La problématique de la conduite des irrigations est ici très différente de celle concernant les cultures à fortes marges brutes. Cela tient au fait que le coût relatif de l'irrigation est élevé par rapport au produit de la culture.

Ainsi dans le cadre d'aménagement collectif, la charge d'irrigation à l'hectare peut se décomposer en $1300 \mathrm{~F}$ pour l'infrastructure, $600 \mathrm{~F}$ pour le matériel et $400 \mathrm{~F}$ pour le fonctionnement, soit au total $2300 \mathrm{~F}$ par hectare.

En fait, on observe des coûts pour de nouveaux irrigants pouvant varier de 1500 à $3000 \mathrm{~F} /$ ha (B. BALAS et al., 1989). 
Cela représente de 12 à $25 \%$ du produit brut avec une part importante due à l'infrastructure et au matériel $(80 \%)$ par rapport au fonctionnement $(20 \%)$. Il faut s'attendre avec la nouvelle PAC à une augmentation relative de la charge d'irrigation (15 à $30 \%$ du produit brut).

La nécessité de limiter les charges d'infrastructure conduit tout aménageur à fixer une limite d'équipement. Le seuil de $80 \%$ de couverture des besoins de pointe est généralement retenu. Il constitue une contrainte d'autant plus grande que l'agriculteur tend vers la monoculture de plantes estivales exigeantes à la période des plus fortes ETP. Par ailleurs, l'agriculteur ne prend que difficilement en compte cet aspect du problème. Il est souvent tenté après quelques années de demande climatique modérée, d'augmenter inconsidérément ses surfaces irriguées sans s'assurer du risque qu'il encourt.

L'incertitude des ressources en eau (limitation due aux hivers secs, des volumes dans le cas des lacs, des débits dans le cas des nappes) a tendance à accentuer cet état de fait.

En effet, dans ce contexte, ce sont les cultures spéciales qui sont prioritaires et l'agriculteur va mobiliser les moyens nécessaires pour couvrir leurs besoins sans risque; ceci au détriment des grandes cultures qui auront à leur disposition un volume et (ou) un débit limité.

La gestion de l'irrigation des grandes cultures sera donc très dépendante des choix stratégiques pris avant la période d'irrigation (Peyremorte P., Tron G., 1989; Balas B., DEUMIER J.M., 1989) à savoir :

- le choix de l'assolement sur la sole irrigable par rapport aux contraintes de ressources en eau, de matériel et de main-d'œuvre ;

- la réflexion sur un calendrier prévisionnel d'arrosage précisant la répartition des volumes et des débits :

- par espèce en fonction des objectifs de rendement et des priorités entre espèces ;

- dans le temps, en tenant compte du niveau d'alimentation hydrique, éventuellement de rationnement de chacune des espèces.

Cette prévision est absolument indispensable pour la conduite des irrigations dans la mesure où elle permet de tenir compte des contraintes de ressources et de distribution de l'eau; elle peut conduire à des décisions justifiées mais surprenantes pour le néophyte:

- irriguer très tôt en saison alors que les plantes ne souffrent pas dans le cas de débit limité ;

- différer le début des irrigations alors que les plantes sont stressées dans le cas de volume limité.

Des outils existent pour aider l'agriculteur à prendre ces décisions.

LORA - logiciel d'optimisation et de recherche d'assolement - (LEROY, JACQUIN, 1991) permet de rechercher l'assolement optimal en tenant compte du milieu (réserve en eau du sol et historique climatique), des contraintes de ressources, de matériel et de main-d'œuvre et en intégrant bien sur les prix des produits et les charges.

Après plusieurs optimisations, en faisant varier certaines hypothèses, on a une idée précise de l'assolement optimal et des volumes d'eau disponibles pour chacune des espè- ces; on peut alors élaborer une stratégie d'eau par espèce et interespèces.

La méthode du «BILANEAUMETRE », récemment explicitée sous le nom de MIRA (méthode d'irrigation raisonnée) développée par la Société du Canal de Provence (Peyremorte P., 1986, 1992) qui donne un calendrier prévisionnel des besoins optimisés d'arrosage établi pour chaque culture sur la base de considérations d'ordre stratégique.

Cette méthode comporte d'une part un logiciel de programmation prévisionnelle des irrigations (OPTIMIRR) qui permet de définir la courbe des besoins périodiques d'arrosage de pointe, optimisés selon divers critères liés à l'objectif de rendement visé et à la stratégie d'irrigation retenue.

Les besoins optimaux d'arrosage se définissent soit en fonction de paramètres physiologiques ETopt périodique selon les cultures - cas des légumineuses, comme le pois (Deumier J.M., 1992), cas du tournesol (Merrien A., 1992) - soit des paramètres économiques, ETopt dépendant alors de l'optimum économique de rendement.

Cette méthode insiste aussi sur l'intérêt de gérer la réserve mobilisable d'eau du sol pour limiter les besoins d'équipement. D'autre part, elle présente ses résultats sous forme d'un tableau de bord graphique désigné EQUILHYDRE.

Ce tableau permet de tenir le bilan effectif des apports d'eau en fonction de la pluviométrie réellement constatée chaque année, variable la plus conséquente des besoins d'irrigation.

\section{Les outils d'aide à la décision pour les choix tactiques et leurs limites}

L'importance des choix stratégiques étant posée, examinons maintenant les outils d'aide à la décision pendant la campagne d'irrigation. Notons en préliminaire que ces moyens ne peuvent en général permettre que de réduire les quantités d'eau initialement envisagées sur la base de considérations d'ordre stratégique, par exemple lorsque les pluies sont nettement plus importantes que d'ordinaire ou encore lorsque la contribution potentielle du sol a été mal appréciée. Très généralement en grande culture, les moyens d'arrosage sont le facteur limitant et les informations susceptibles de vérifier une augmentation de la demande deviennent superflues.

Parmi les outils d'aide à la décision :

- Le bilan hydrique est le plus connu et le plus diffusé par différents organismes. Son principe est d'estimer un déficit d'eau dans le sol pour piloter les irrigations.

D'un point de vue strictement technique, il est intéressant de se demander dans quelle mesure le bilan hydrique permet de rendre compte :

- de la consommation en eau du végétal ;

— de l'évolution du stock d'eau dans le sol.

Le résultat est influencé par la connaissance des termes du bilan. 
Ne serait-ce qu'en matière d'estimation de l'ETM, il a été démontré pour le maïs (PEYREMORTE et RosSET, 1977) que l'intervalle de prédiction à partir de l'ETP est de $\pm 1,5$ à $1,9 \mathrm{~mm} /$ jour pour des ETM de 5 à $8 \mathrm{~mm} /$ jour. La précision des coefficients $\mathrm{k}$ est donc très médiocre.

Une incertitude très importante demeure sur l'estimation annuelle des contributions en eau du sol. Les agronomes évaluent les contributions en eau potentielles. Par contre, chaque année, on doit s'interroger sur l'état de remplissage des sols. Nous avons connu ces dernières années des réserves non reconstituées dans des régions où habituellement elles le sont (cas de Midi-Pyrénées et de la région Centre). Les tensiomètres sont alors extrêmement utiles pour corriger ces informations même s'ils ne permettent pas de quantifier la réserve restante dans le sol.

D'autre part, dans le cas de sol à nappes et dans le cas de sol profond, il peut exister des remontées capillaires non négligeables (TARDIEU, 1991) non prises en compte par les modèles simplifiés de bilan hydrique. Il est important alors, de repérer ce type de situation ce qui n'est pas toujours le cas actuellement. L'Opération des Secteurs de Références Irrigation pourrait inciter à mettre au point une démarche dans le cadre des études pédologiques.

Par ailleurs pour son application, le bilan hydrique impose la connaissance des doses d'irrigation. Or, il s'avère que bon nombre d'irrigants apprécient mal les doses pratiquées, essentiellement à cause des difficultés de réglage des matériels, particulièrement des enrouleurs.

Les résultats des opérations diagnostics (DUBALEN, 1992) montrent que dans $46 \%$ des cas pour les enrouleurs, dans $34 \%$ des cas pour la couverture intégrale, la dose contrôlée diffère de plus de $20 \%$ de la dose annoncée par l'agriculteur.

Au-delà de ces problèmes techniques, la mise en œuvre du bilan hydrique par les agriculteurs, devrait s'accompagner d'une réflexion préalable sur la gestion stratégique des moyens d'arrosage (dont la réserve en eau des sols). Il est bien évident que sans cette réflexion, l'utilisation du bilan ne peut être qu'inopérante.

- La mesure de la teneur en eau du sol à l'aide d'une sonde à neutrons est une méthode fiable. Des services réalisant, à titre onéreux, des campagnes de mesure existent dans certains pays (USA, Australie) en vue d'améliorer les pratiques d'irrigations chez les exploitants.

En France l'usage de la sonde à neutrons reste le fait d'équipes spécialisées. Il s'agit d'une méthode « lourde » au plan pratique; ... coût de l'équipement et de sa maintenance pour assurer l'étalonnage et l'échantillonnage afin de garantir une représentativité acceptable des informations obtenues.

Cette méthode reste cependant la seule disponible pour valider efficacement les multiples hypothèses retenues en matière de fonctionnement hydrique des sols. Cette étape demeure indispensable pour adapter et rendre crédible les méthodes reposant sur le bilan hydrique utilisé selon des considérations soit tactiques soit stratégiques.

- Plus largement vulgarisées, les mesures tensiométriques (Peyremorte P., 1983) connaissent un regain d'intérêt avec l'apparition de nouvelles sondes à mesure électrique.
Ces sondes présentent l'avantage de ne plus comporter, contrairement aux tensiomètres classiques, de circuit hydraulique.

Ainsi, elles sont d'un emploi plus facile. De plus, elles sont capables de mesurer dans une gamme de 10 à 200 centibars.

Comparativement à des tensiomètres, elles se sont avérées donner des informations identiques au-dessus de 10 centibars (Peyremorte P., Tron G., 1991) ; par contre, en-dessous de cette valeur, les mesures ne sont pas significatives, ce qui ne permet pas d'analyser les situations de très forte teneur en eau (engorgement). De plus, la mesure simultanée de la température à la profondeur analysée est indispensable.

Ce type de mesure étant automatisable à partir de diverses centrales d'acquisition, on peut imaginer qu'il connaisse un bon développement. D'ores et déjà, l'appareil HUMICRO 2000, dont il a été question précédemment, peut constituer un concours précieux pour prendre des décisions de conduite d'arrosage. La présentation des mesures de 8 capteurs faite quotidiennement sous forme graphique offre une facilité remarquable d'exploitation des données.

Toutefois, ce progrès relatif à l'acquisition des mesures tensiométriques ne doit pas cacher la complexité d'utilisation pratique. Seules les personnes capables de formuler des hypothèses et de les vérifier par ces mesures, sont à même d'en tirer le plus grand profit.

- Les indicateurs de stress hydrique "plantes" sont encore du domaine de la recherche

Dans le but de mieux valoriser l'eau et de piloter les irrigations en fonction des besoins réels, les chercheurs mettent au point des indicateurs mesurés directement sur les plantes. Il s'agit de prendre la plante comme révélateur synthétique qui intègre les différents éléments du fonctionnement hydrique du système sol-plante-atmosphère.

La mesure des variations micrométriques de diamètre des tiges, possible par l'emploi de PEPISTA, constitue un élément de diagnostic très intéressant d'autant que ce type de mesure est automatisable. Il en a été déjà question au premier paragraphe. Cependant l'utilisation de ce matériel pose encore divers problèmes en grandes cultures :

- le nombre de points de mesure,

- la faisabilité des mesures selon le support végétal,

- l'interprétation des données.

La température de surface du couvert est un indicateur de son état hydrique. Les mesures sont possibles à l'aide de radiothermomètre portable à partir d'un indice foliaire de 2 environ, moyennant certaines précautions. L'idéal est une journée à rayonnement stable et peu ventée. Un certain nombre d'indices thermiques peuvent être calculés à partir de la température de surface du couvert.

Le Stress Degree Day, cumul des écarts entre la température de surface Ts et la température de l'air Ta, est un indice intéressant et assez facile à obtenir. Le cumul des valeurs positives de Ts-Ta permet de s'affranchir des variations journalières de Ts dues aux variations climatiques. De nombreuses difficultés subsistent, en particulier: 
- l'utilisation avant que la culture ne couvre totalement le sol :

- la détermination d'un seuil de déclenchement des irrigations valable quel que soit le scénario climatique ;

- comment prendre en compte les pluies?

— comment intégrer les journées moyennes et ventées où la mesure est difficile?

(Ruelle et al., 1992; Deumier et al., 1992).

Il existe un indice plus élaboré le Crop Water Stress Index dont la valeur correspond théoriquement à 1 -(ETR/ETM). Son calcul nécessite de disposer de données météorologiques complexes (le rayonnement, l'humidité de l'air et la température de l'air...) ou le calage de fonctions empiriques. Les résultats semblent assez décevants sous nos climats : variabilité climatique pendant la mesure, gamme de déficit de pression de vapeur faible.

Un autre indicateur souvent utilisé à titre expérimental est le potentiel hydrique foliaire : le potentiel hydrique de base, mesuré avant le lever du jour, est considéré comme le plus intéressant, car représentant un état d'équilibre de l'eau entre le sol et la plante après quelques heures sans transpiration importante (N. KATERJ et al., 1988).
Les auteurs signalent toutefois certaines situations où le potentiel de base est inefficace ; par exemple lors de remontées capillaires venant du sol sous le niveau d'enracinement (TARDIEU et al., 1991) ou de plante à enracinement profond comme le sorgho cultivé en sol profond (RUELLE et al., 1992).

Le potentiel hydrique à midi solaire est plus difficile à interpréter notamment pour les plantes à comportement isohydrique (N. KATERJ et al., 1988).

Indépendamment des problèmes d'interprétation des valeurs, le frein essentiel au développement au champ de cette technique est qu'elle n'est pas automatisable et que la mesure ne peut être réalisée que sur un échantillon de faible taille.

Les trois indicateurs pris ici en exemple: la mesure micrométrique, la température du couvert et le potentiel foliaire, peuvent être utilisés pour expliquer des comportements lors d'expérimentation agronomique et pour caler des outils de pilotage plus opérationnels chez l'agriculteur (bilan, tensiomètre). Il est encore trop tôt pour qu'ils soient directement utilisés en exploitation agricole.

\section{Conclusion}

Un grand nombre de techniques se présentent actuellement comme susceptibles de contribuer à l'amélioration de la maîtrise des arrosages.

L'utilité de certaines d'entre-elles restera au bénéfice du progrès de la connaissance des mécanismes régissant la réaction des cultures au stress hydrique. Jusqu'alors la plus grande difficulté était de pouvoir apprécier avec suffisamment de précision de déficit d'ET réellement engendré par une restriction imposée afin d'en interpréter correctement les conséquences.

Les procédés basés sur des mesures automatiques pourront prendre une place dans le pilotage des irrigations, soit directement par l'intermédiaire d'un automate, soit indirectement en fournissant des informations facilement accessibles, voire pré-interprétées.

Cependant, dans ce cas, ces procédés tendront à assurer une conduite des apports en temps réel qui ne peut qu'entraîner une augmentation des besoins de pointe et donc des moyens d'arrosage afférents. Cela peut être parfaitement acceptable pour des cultures à forte marge.

Par contre, les grandes cultures sont tributaires d'une autre logique davantage orientée sur la gestion des moyens de production qui devient prépondérante sur le pilotage proprement dit.

Comme nous l'avons indiqué avec insistance, les besoins instantanés des cultures doivent alors laisser la place à des stratégies concernant des aspects plus économiques que techniques.

Il n'en reste pas moins que les moyens de mesures utilisables in situ, auront toujours une utilité pour valider les méthodes de conduite basées sur des concepts d'ordre statistique. Par ailleurs, leur utilité existe aussi pour limiter les arrosages chaque fois que l'estimation des besoins s'avère excessive notamment par suite d'une contribution des réserves du sol supérieure à celle calculée.

On comprend donc l'intérêt d'associer diverses méthodes pour garantir une plus grande maîtrise de l'usage de l'eau et des moyens nécessaires à sa mise en œuvre. 


\section{Bibliographie}

Deumier J.M., Bouthier A. (1992). - Water stress effect during pea preflowering period, Recueil de communications de la première Conférence Européenne sur les Protéagineux, p. 281-282.

Deumier J.M., Duschesne J., Bouthier A., Rapanoelina M. (1992). - Détermination du stress hydrique du blé par radiothermométrie infrarouge, Perspectives Agricoles, Novembre 1992, 8 p.

Dubalen J. (1992). - Problèmes rencontrés par les irrigants en matière de réglage et d'utilisation des matériels d'irrigation, L'irrigation en Grandes Cultures, Chambre Régionale d'Agriculture de Midi-Pyrénées, $17 \mathrm{p}$.

Huguet J.G. (1992). - Pepista : Programmateur d'irrigation par les micro variation du diamètre de la plante - Journées CIID - $16^{e}$ Conférence Européenne BUDAPEST, juin $1992,10 \mathrm{p}$.

Merrien A. (1992). - L'irrigation du tournesol, dans l'irrigation en Grandes Cultures, Recueil des communications de la journée du 9 avril 1992 - Chambre Régionale d'Agriculture de Midi-Pyrénées, $5 \mathrm{p}$.

Peyremorte P. (1992). - Quels outils pour programmer l'irrigation des grandes cultures?, Lettre d'information de la Société du Canal de Provence et d'Aménagement de la Région Provençale $n^{\circ} 5,3$ p.

Peyremorte P., Chol P. (1992). - Pilotage automatique optimisé des irrigations localisées - Journées CIID - $16^{e}$ Conférence Européenne, Budapest, juin 1992, 12 p.

Ruelle P., BAldy Ch. (1992). - Utilisation d'indicateurs d'état hydrique pour la conduite d'une culture - Cas du soja et du sorgho - I.C.I.D. 16th European Regional Conference - 21-27 juin 1992 - Budapest.

Berdou C. (1991). - Validation des méthodes de pilotage de l'irrigation en grande culture : cas du soja, Mémoire DAA ENSAM, CEMAGREF, $77 \mathrm{p}$.

LEROY P., JACQUIN C. (1991), - LORA : a decision support system for the choice of crops on the irrigable area of a farm, " dans acte du Colloque " décision support systems : ifors-spc 1, Bruges, March 26-29, 1991.

Olufayo A., 1991. - Etude comparée de plusieurs indicateurs de stress hydrique du sorgho grain - Mémoire DAA ENSAR, INRA LECSA, $65 \mathrm{p}$.
Peyremorte P., Tron G. (1991). - Etude du fonctionnement des sondes tensiométriques Irrometer WATERMARK, $15 \mathrm{p}$.

TARdieu F., Katerji N., Bethenod O. (1991). - Réponses de plusieurs indicateurs de l'état hydrique du maïs à la réserve en eau du sol, dans Gestion de l'eau dans les systèmes de production de grandes cultures du Sud-Ouest de la France, Convention Ministère de la Recherche et de la Technologie - n ${ }^{\circ} 88 \mathrm{G} 0631$, p. 172-181.

Peyremorte P. (1990). - Nécessité de connaître l'état et le fonctionnement hydrique du sol pour gérer convenablement l'irrigation - Journées scientifiques $G F H N, 11$ et 12 décembre $1990,17 \mathrm{p}$.

Balas B., Deumier J.M. (1989). - " Gestion de l'eau au niveau de l'exploitation agricole". Colloque sur les méthodes d'irrigation améliorées pour préserver et protéger les ressources en eau et augmenter le rendement des cultures. Nations Unies, $16 \mathrm{p}$.

Peyremorte P., Tron G. (1989), - Prédominance du contexte physique et humain pour le choix d'une stratégie de pilotage des irrigations en grandes cultures - Colloque international sur les méthodes améliorées d'irrigation, Avignon 25-29 septembre 1989, 12 p.

Katerji N., Itier B., Ferreira I. (1988). - Etude de quelques indicateurs de l'état hydrique d'une culture de tomates en région semi-aride, Agronomie, 8 (S), p. 425-433.

Peyremorte P. (1986). - Simplification de l'estimation approximative des besoins d'arrosage : le bilaneaumètre - Eau et aménagement de la Région Provençale, $\mathrm{n}^{\circ} 43,8 \mathrm{p}$.

Peyremorte P. (1984). - Pilotage des irrigations: adéquation des estimations ou des mesures - Bulletin $G F H N, \mathrm{n}^{\circ} 16$, $36 \mathrm{p}$.

Peyremorte P. (1983). - Des tensiomètres pour améliorer la conduite des arrosages - Perspectives Agricoles, $\mathrm{n}^{\circ} 67$.

Peyremorte P., Rosset J.M. (1977). - Variabilité des coefficients culturaux $\mathrm{K}-$ Conséquences pour la définition d'une méthode d'irrigation basée sur le concept d'ETP, $\mathrm{La}$ météorologie, $\mathrm{VI}^{\mathrm{e}}$ Série $\mathrm{n}^{\circ} 11$, p. 61-68. 